\title{
Epidural spinal cord stimulation for recovery from spinal cord injury: its place in therapy
}

\author{
This article was published in the following Dove Press journal: \\ Journal of Neurorestoratology \\ 19 September 2016 \\ Number of times this article has been viewed
}

\section{Line Jacques \\ Michael Safaee \\ Department of Neurological Surgery, University of California, San Francisco, San Francisco, CA, USA}

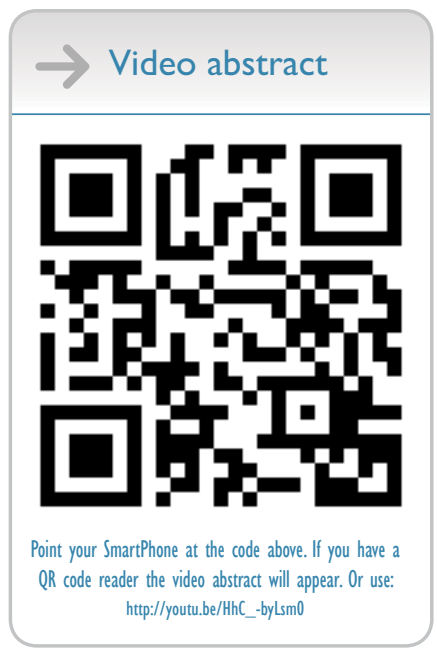

Correspondence: Line Jacques Chief of Peripheral Nerve and Pain Surgery, University of California, San Francisco, 505 Parnassus Avenue, Room M779 San Francisco, CA 94143, USA

Tel +l 4I5 3537500

Fax + I 4153532889

Email line.Jacques@ucsf.edu
Abstract: This paper is a review of some of the current research focused on using existing epidural spinal cord stimulation technologies in establishing the effectiveness in the recovery of independent standing, ambulation, or intentional movement of spinal cord injury patients. From a clinician's perspective, the results have been intriguing, from a restorative perspective they are promising, and from a patient's perspective they are hopeful. The outcomes, although still in the experimental phase, show some proof of theory and support further research. From a high volume university based clinician's perspective, the resources needed to integrate this type of restorative care into a busy clinical practice are highly challenging without a well-structured and resource rich institutional restorative program. Patient selection is profoundly critical due to the extraordinary resources needed, and the level of motivation required to participate in such an intense and arduous rehabilitation process. Establishing an algorithmic approach to patient selection and treatment will be paramount to effectively utilize scarce resources and optimize outcomes. Further research is warranted, and the development of dedicated technological hardware and software for this therapeutic treatment versus using traditional spinal cord stimulation devices may yield more robust and efficacious outcomes.

Keywords: independent standing, ambulation, intentional movement, recovery, rehabilitation, locomotion

\section{Introduction}

Few conditions cause the degree of physical, psychological, and emotional consequences as those associated with spinal cord injury. Recovery from spinal cord injury is rare and most patients are left with permanent disability, which often has devastating consequences on every aspect of their daily activities. There are an estimated 300,000 patients living with spinal cord injury in North America with an additional 20,000 new injuries every year. ${ }^{1,2}$ The cost of these injuries, including treatment and rehabilitation, is estimated at US\$9.7 billion dollars per year in the United States alone. ${ }^{1}$

Complete spinal cord injury destroys neural circuitry within the spinal gray matter and shears long white matter tracts resulting in loss of function below the level of the lesion. ${ }^{3}$ Clinical recovery from complete spinal cord injury is poor, with limited therapeutic options for patients. Standard-of-care rehabilitation has limited efficacy and is unable to restore the ability for independent standing, ambulation, or intentional movements. ${ }^{4}$ There is no effective therapy for regeneration of voluntary motor function and thus medical care for these patients instead focuses on prevention of secondary injury and complications from spinal cord injury and chronic immobility. 
Intense physio and occupational therapy is often employed in a rehabilitation environment, along with physiatry services to aid in assessment and treatment of the spinal cord injured patient. Ultimately, the goal of these treatments is to provide some functional improvements, which may allow some dignified self-sufficiency, keeping in mind the extent and site of their injury. Complaints of bladder, bowel, and sexual dysfunction along with temperature dysregulation are common and provoke high anxiety in patients suffering from spinal cord injury.

Emerging data suggest that epidural spinal cord stimulation may have a role in restoring some motor control functions used for standing, walking, or limb movement through sensory input and act as a spinal circuitry controller for these functions.

\section{Background}

Epidural spinal stimulation was initially used to treat intractable pain; however, its use expanded following an unexpected improvement in motor function in a patient with multiple sclerosis. ${ }^{5}$ Current systems consist of two primary types of lead systems. The most common being a cylindrical array of electrodes bonded to a polyurethane lead body creating a circumferential electrical field, which consists of either eight or 16 contacts per lead with a fixed geometrical arrangement. The geometrical design provides a defined contact size in the longitudinal direction along with a defined spacing between individual electrode contacts. This geometry is used to control the stimulating electrical field that is generated by using combinations of these electrodes in an anode or cathode arrangement. Surgical leads are less frequently used due to the added complexity of performing a laminotomy/ laminectomy and therefore restricting implantation to certain surgical subspecialties. Surgical leads provide many benefits in electrical field steering, by maintaining a distinct geometry in both the longitudinal and horizontal directions. This allows for greater reproducibility of electrical field stimulation patterns, even if the lead migrates in any given direction. It also allows for multiple columns of geometrically defined electrical contacts in both longitudinal and horizontal directions with precise spacing within those directions. The array is then connected to an implanted pulse generator or remotely coupled via radiofrequency to an external pulse generator. Electrode contacts may be arranged with single or multiple anodes or cathodes, depending on the complexity of the pain patterns being treated and the sophistication of the device. Ultimately, the goal is to fine-tune the electrical field to provide the most adequate pain coverage based on the individual patients needs. ${ }^{6}$ However, in this subset of patients (spinal cord injured patients), pain management is not the intended outcome, instead ultimately, the goal is to re-establish neural pathways, which will induce willful and controlled motor function in spinal cord injured patients.

\section{Treatment of paralysis}

Epidural spinal cord stimulation for the treatment of spinal cord injury is an emerging treatment opportunity, which is still in its infancy. Although there has been some early work in demonstrating the ability to aid in standing, walking, and limb movement, the number of patients having undergone this experimental treatment is still insufficient to draw any long-term therapeutic outcome conclusions. In the treatment of lower limb paralysis, electrodes are placed more caudally (T11-L1, at the lumbosacral spinal cord) compared to those used to treat pain (T8-T10) or spasticity. The role of stimulating dorsal roots in generating motor responses is not fully understood aside from orthodromic inflow generated in the afferent axons within the posterior roots and antidromic transmission of action potentials to synaptic terminals. ${ }^{7}$ An early study in six patients with complete spinal cord injury showed that epidural stimulation was able to generate rhythmic, locomotor-like activity in the lower limbs. ${ }^{6}$ Subsequent studies showed that lumbar spinal cord circuitries were capable of converting tonic signals into coordinated oscillating motor outputs, consistent with an intrinsic pattern generator. ${ }^{8,9}$

Most recently, data have emerged showing return of voluntary function after spinal cord injury with epidural stimulation. Angeli et al presented results of four patients with chronic complete motor paralysis to execute voluntary tasks after presence of epidural stimulation. ${ }^{10}$ In this study, an epidural spinal cord stimulator (Medtronic, RestoreAdvanced) and 16-electrode array were implanted at vertebrae T11-T12 over the spinal cord segments corresponding to L1-S1 in four patients with motor complete spinal cord injury. All patients were medically stable without cardiopulmonary disease or dysautonomia that could contraindicate standing or walking with support, they had no painful musculoskeletal dysfunction, unhealed fractures, contractures, pressure sores, or urinary tract infections. The patients also had to be cleared of any significant depression or drug abuse, be free of anti-spasticity medication regimens, and lack progressive spinal cord injury above T10. None of these patients had motor responses present in leg muscles during transcranial magnetic stimulation and no volitional control during voluntary movement attempts in leg muscles when measured by electromyography (EMG). All individuals in the study 
were males at least 2 years from the date of initial injury with levels ranging from C7-T5. The average age was 26 years, and all patients had failed physical therapy and were unable to stand or walk independently. All four individuals were able to execute intentional movements of the legs in response to verbal command, with epidural stimulation present. All patients were able to generate EMG activity and movement during ankle dorsiflexion in the presence of epidural stimulation. The authors also assessed whether visual and auditory input could modulate the level of activation. When asked to synchronize their movement to rise and fall of a sine wave on a computer screen, individuals were able to consistently activate the appropriate muscles for the specified action. These results demonstrated that auditory and visual cues were processed by the sensorimotor cortex such that the appropriate spinal interneurons below the level of injury were able to titrate the desired level of excitability of the desired motor pools. In two of the four patients included in the study, daily training with epidural stimulation with stand training and home-based voluntary training resulted in the generation of voluntary efforts with higher forces and lower stimulation voltages. Several key findings should be emphasized from this study. In three individuals, testing of epidural stimulation after implantation, but before repetitive training suggests that descending connections may have existed since the time of injury. The inability of transcranial magnetic stimulation to show any excitation event suggested that the alteration of spinal circuitry from epidural stimulation was the enhancement of the central excitatory drive to the motor neurons. The authors propose that the functional state and excitability of spinal interneurons and motor neurons were modulated by epidural stimulation, thus driving them closer to an appropriate activation threshold. Additionally, the improvement in voluntary movements over time with daily epidural stimulation demonstrated the ability of spinal networks to learn from task-specific training and improve motor pool recruitment. Most importantly, this study showed that individuals with complete motor paralysis can develop functional connectivity across the site of injury in the presence of epidural stimulation.

Perhaps some of the most intriguing results came from a study by Harkema et al, which looked at a single patient case study of a 23-year-old male involved in a motor vehicle accident 3 years prior to implantation. ${ }^{11}$ Neurological examination revealed paraplegia from $\mathrm{C} 7-\mathrm{T} 1$ subluxation with injury to the lower cervical and upper thoracic spinal cord. He had no contraction of the trunk or leg muscles. The triceps and intrinsic hand muscles exhibited weak voluntary contraction, and had no contraction of the trunk or leg muscles. After postoperative care, the magnetic resonance imaging (MRI) revealed myelomalacia and atrophy of the cord segment adjacent to the $\mathrm{T} 1$ vertebral body. The patient received 170 locomotor training sessions prior to epidural stimulation implantation, followed by 29 stimulation experiments. These experiments demonstrated that continuous stimulation modulated the physiological state of the spinal cord, which enabled sensory information processing that was closely linked to the functional task. Even more intriguing to the researchers were the changes in the autonomic function of the bladder, sexual, and thermoregulatory activity, which was perceived to be a significant benefit to the patient. ${ }^{11}$

\section{Treatment of spasticity}

An unfortunate consequence of spinal cord injury is spasticity, which leads to a disequilibrium in muscle tone balance of the limbs and can result in debilitating contractures. Spasticity can mask residual motor function, limit passive and active movements, and result in discomfort and pain. Neurosurgical treatments for spasticity are either destructive or neuromodulatory in nature. ${ }^{12}$ Destructive treatments are performed with the goal of interrupting the stretch reflex through lesioning the posterior horn (dorsal root entry zone), posterior rootlets (selective dorsal rhizotomy), or peripheral nerve (selective peripheral neurotomy). These operations can reduce spasticity but have the risk of inducing muscle weakness and neuropathic pain. Neuromodulation does not require neural lesioning thus possesses a safer complication profile, with the option of reversibility should newer treatment options emerge. Modulatory approaches include intrathecal baclofen and chronic spinal cord stimulation. The exact mechanisms underlying spasticity in these patients are not fully understood but thought to be secondary to a reduction in segmental inhibition. ${ }^{13,14}$ Early reports of epidural stimulation for treatment of spasticity date back to the 1970s and 1980s. ${ }^{15-17}$ In a large series of patients with both traumatic and nontraumatic myelopathy, epidural stimulation showed a reduction in spasm severity but high complication rates, including infection, electrode migration, and hardware failure. ${ }^{18}$ More recent studies have argued that epidural stimulation for patients with spinal cord injury-related spasticity lacks long term efficacy and cost-effectiveness. ${ }^{19}$ The use of intrathecal baclofen provides the best overall control and management of spasticity due to spinal cord injury. The titratable benefits of baclofen therapy may allow some patients to have dose control associated with individual activities and provide adequate spasticity control with minimal loss of 
coordinated muscle function, providing satisfactory ambulation in incomplete lower limb spinal cord injured patients.

\section{Treatment of pain}

Shealy et al were the first to treat intractable pain with epidural stimulation in $1967 .{ }^{20}$ Since then, there have been many animal studies focused on mechanisms of action of epidural spinal cord stimulation and pain suppression. ${ }^{21-25}$ Modern variations of this therapy consist of electrode insertion into the epidural space followed by a trial period of 2-7 days to determine if patients will have a $50 \%$ or greater reduction in their pain symptoms and to determine how they will tolerate neuromodulation as a therapeutic treatment. Once a decision to proceed is made, a pulse generator is implanted in the subcutaneous space and all hardware are internalized. The mechanisms underlying pain alleviation are not clearly understood but believed to stimulate dorsal column fibers that inhibit nociceptive impulses; however, others believe the effects are more widespread and may include modulation of neurotransmitter release. ${ }^{7,26}$ The procedure is safe with a relatively low complication rate. ${ }^{27}$ Several studies have assessed the efficacy of this therapy for patients with complex regional pain syndrome and failed back surgery syndrome. ${ }^{28-31}$ Epidural stimulation has also shown positive improvements in treating peripheral vascular disease and intractable angina by causing vasodilation and improved small vessel flow. Most notably, when using spinal cord stimulation for the treatment of refractory pain, paresthesia is created to mask the pain symptoms of the patient and provide a more pleasant sensory experience relative to their unsolicited pain. This is in stark contrast to the use of epidural stimulation for the treatment of spinal cord injury requiring significantly higher stimulation parameters, which activate the dorsal root fibers and initiate motor function. Typically this level of stimulation would be deemed unpleasant by a patient being treated for their pain symptoms vs ambulation assistance (personal experience).

\section{Conclusion}

Epidural spinal cord stimulation has proven to be effective in the treatment of chronic refractory pain and many other pain-related disorders. Early results in the use of epidural spinal cord stimulation, shows some novel improvements in patients with spinal cord injury. Patient selection and counseling are of critical importance. The number of patients treated to date is few and the results are heavily dependent on a large interdisciplinary team to assess and treat the patient. It would be difficult to incorporate this treatment option into a clinical setting without being closely affiliated with an extensive series of experts trained in rehabilitative medicine. One could assume that outcomes may vary considerably based on the type of spinal cord injury (anatomical location, complete or incomplete), age of the patient, motivation for improvement, and the ability to be engaged in a long-term rehabilitative treatment plan. There is a large pool of young, healthy, and motivated spinal cord injured military personnel, who may provide exceptional candidacy and willingness for evaluating the effectiveness and benefits of this adolescent therapeutic modality. Considering that epidural spinal cord stimulation has been accepted as a primary treatment for chronic refractory pain, the efficacy and risks have been well documented and this therapy has emerged to be a relatively safe therapeutic modality. Extrapolating those same benefits and risks to an appropriately selected group of spinal cord injury patients, along with a hyper vigilant and intense rehabilitation program, would help provide better comprehensive outcome measures to evaluate the benefits toward a larger population of patients.

Emerging data demonstrating return of motor function after complete paralysis is hopeful and certainly within the desires of most spinal cord injury patients. Although, this treatment still has to be viewed as experimental for this application and may be a single treatment in a comprehensive conjunctive treatment continuum. We have observed patients with other neuromodulatory managed conditions requiring conjunctive therapies to manage the more comprehensive nature of their symptoms. There is a strong probability that this may be the case in spinal cord injury, considering the complex nature of their condition and the extent of their neurophysiological dysfunction. Lastly, with the advancements in medical imaging and diagnostics, such as diffusion tensor imaging, tractography etc and the advent of MRI compatible neuromodulation hardware, we have the opportunity to gain a better understanding of the real physiological mechanisms of action associated with these treatments, and how they may provide benefit in patients with these complex injuries allowing us to forge unique treatment plans specific to the patient's needs. One must clearly define the logistics of offering these types of procedures for this application, based on their institutions level of sustainable engagement, ie, clinical vs academic vs research. Considering that the majority of epidural spinal cord stimulation devices implanted in the United States are administered in an outpatient clinical setting, their resources would be insufficient to provide the level of rehabilitation necessary to optimize this treatment for spinal cord injury. Even within our own academic center, we lack the resources to engage in such a complex rehabilitation program in this 
subset of patients. The motivation, drive, and tenacity required to undertake such an exhaustive rehabilitation process would preclude many spinal cord injury patients from candidacy.

\section{Disclosure}

The authors report no conflicts of interest in this work.

\section{References}

1. Ackery A, Tator C, Krassioukov A. A global perspective on spinal cord injury epidemiology. J Neurotrauma. 2004; 21(10): 1355-1370.

2. Fehlings MG, Tighe A. Spinal cord injury: the promise of translational research. Neurosurg Focus. 2008; 25(5): E1.

3. Kakulas BA, Kaelan C. The neuropathological foundations for the restorative neurology of spinal cord injury. Clin Neurol Neurosurg. 2015;129(1): S1-7.

4. Dietz V, Fouad K. Restoration of sensorimotor functions after spinal cord injury. Brain. 2014; 137(3): 654-667.

5. Cook AW, Weinstein SP. Chronic dorsal column stimulation in multiple sclerosis. Preliminary report. NY State J Med. 1973. 73(24): p. 2868-72.

6. Dimitrijevic, MR, Gerasimenko Y, Pinter MM. Evidence for a spinal central pattern generator in humans. Ann NY Acad Sci. 1998;860: 360-376.

7. Tator $\mathrm{CH}$, Minassian K, Mushahwar VK. Spinal cord stimulation: therapeutic benefits and movement generation after spinal cord injury. Handb Clin Neurol. 2012;109: 283-296.

8. Gerasimenko YP, Makarovskii AN, Nikitin OA. Control of locomotor activity in humans and animals in the absence of supraspinal influences. Neurosci Behav Physiol. 2002; 32(4): 417-423.

9. Pearson K, Gordon J, Locomotion. In: Principles of neural science. Eds. Kandel ER, Schwartz JH, and Jessell TM. 2000, New York: McGraw-Hill.

10. Angeli CA, Edgerton VR, Gerasimenko YP, and Harkema SJ. Altering spinal cord excitability enables voluntary movements after chronic complete paralysis in humans. Brain. 2014;137(5):1394-1409.

11. Harkema S. Effect of epidural stimulation of the lumbosacral spinal cord on voluntary movement, standing and assisted stepping after motor complete paraplegia: a case study. Lancet. 2011;377(9781): 1938-1947.

12. Dekopov AV, Shabalov VA, tomsky AA, Hit MV, Salova. Chronic spinal cord stimulation in the treatment of cerebral and spinal spasticity. Stereotact Funct Neurosurg. 2015: 93(2):133-139.

13. Adams MM, Hicks HL. Spasticity after spinal cord injury. Spinal Cord. 2005; 43(10): 577-586.

14. Elbasiouny SM, Moroz D, Bakr MM, Mushahwar VK. Management of spasticity after spinal cord injury: current techniques and future directions. Neurorehabil Neural Repair. 2010; 24(1): 23-33.

15. Barolat G. Surgical management of spasticity and spasms in spinal cord injury: an overview. J Am Paraplegia Soc. 1988;11(1): 9-13.

16. Reynolds AF, Oakley JC. High frequency cervical epidural stimulation for spasticity. Appl Neurophysiol. 1982;45(1-2):93-97.
17. Richardson RR, McLone DG. Percutaneous epidural neurostimulation for paraplegic spasticity. Surg Neurol. 1978; 9(3):153-155.

18. Barolat G, Myklebust JB, Wenninger W. Effects of spinal cord stimulation on spasticity and spasms secondary to myelopathy. Appl Neurophysiol. 1988; 51(1): 29-44.

19. Midha M, Schmitt JK. Epidural spinal cord stimulation for the control of spasticity in spinal cord injury patients lacks long-term efficacy and is not cost-effective. Spinal Cord. 1998; 36(3):190-192.

20. Shealy CN, Mortimer JT, Reswick JB. Electrical inhibition of pain by stimulation of the dorsal columns: preliminary clinical report. Anesth Analg. 1967; 46(4): 489-491.

21. Cui JG, Meyerson BA, Sollevi A, Linderoth B. Effect of spinal cord stimulation on tactile hypersensitivity in mononeuropathic rats is potentiated by simultaneous GABA(B) and adenosine receptor activation. Neurosci Lett. 1998; 247(2-3):183-186.

22. Dubuisson D. Effect of dorsal-column stimulation on gelatinosa and marginal neurons of cat spinal cord. J Neurosurg. 1989;70(2): 257-265.

23. Stiller CO, Cui JG, O'Connor WT, Brodin E, Meyerson BA, Linderoth B. Release of gamma-aminobutyric acid in the dorsal horn and suppression of tactile allodynia by spinal cord stimulation in mononeuropathic rats. Neurosurgery. 1996;39(2):367-374.

24. Linderoth B, Gazelius B, Franck J, Brodin E. Dorsal column stimulation induces release of serotonin and substance $\mathrm{P}$ in the cat dorsal horn. Neurosurgery. 1992;31(2):289-296.

25. Myerson BA, Ren B, Herregodts P, Linderoth B. Spinal cord stimulation in animal models of mononeuropathy: effects on the withdrawal response and the flexor reflex. Pain. 1995; 61: 229-243.

26. Linderoth B, Myerson B. Spinal cord stimulation: mechanism of action. In: Surgical management of pain. ed: Burchiel K. 2002, New York: Thieme Medical Publishers.

27. Turner JA, Loeser JD, Deyo RA, Sanders SB. Spinal cord stimulation for patients with failed back surgery syndrome or complex regional pain syndrome: a systematic review of effectiveness and complications. Pain. 2004;108(1-2):137-147.

28. Kemler MA, De Vet HC, Barendse GA, Van Den Wildenberg FA, Van Kleef $M$. The effect of spinal cord stimulation in patients with chronic reflex sympathetic dystrophy: two years' follow-up of the randomized controlled trial. Ann Neurol. 2004;55(1):13-18.

29. North RB, Kidd DH, Farrokhi F, Piantadosi SA. Spinal cord stimulation versus repeated lumbosacral spine surgery for chronic pain: a randomized, controlled trial. Neurosurgery. 2005;56(1):98-106.

30. Simpson EL, Duenas A, Holmes MW, Papaioannou D, Chilcott J. Spinal cord stimulation for chronic pain of neuropathic or ischaemic origin: systematic review and economic evaluation. Health Technol Assess. 2009;13(17): iii, ix-x, 1-154.

31. Taylor RS. Spinal cord stimulation in complex regional pain syndrome and refractory neuropathic back and leg pain/failed back surgery syndrome: results of a systematic review and meta-analysis. J Pain Symptom Manage. 2006;31(4): S13-S19.
Journal of Neurorestoratology

\section{Publish your work in this journal}

The Journal of Neurorestoratology is an international, peer-reviewed, open access online journal publishing original research and review articles on the subject of Neurorestoratology. To provide complete coverage of this revolutionary field the Journal of Neurorestoratology will report on relevant experimental research, technological advances,

\section{Dovepress}

and clinical achievements. The manuscript management system is completely online and includes a very quick and fair peer-review system, which is all easy to use. Visit http://www.dovepress.com/testimonials. php to read real quotes from published authors. 